Case Report

\title{
SPECT-CT imaging in the early diagnosis of osteonecrosis of femoral head after osteosynthesis by percutane screwing in the young adult: a case
} report

\begin{abstract}
We report here the case of a young patient victim of a road accident and having benefited from osteosynthesis by double screwing, in whom the clinical course was marked two years later by the occurrence of osteonecrosis of the femoral head (ONFH) therefore the diagnosis was confirmed by a SPECT CT centered on the pelvis, supplementing a three-phase bone scintigraphy.
\end{abstract}

Keywords: bone scintigraphy, SPECT CT, ONFH
Volume 7 Issue 5 - 2020

\author{
Fabrice Fokoué, Sanae El Mselmi, Nadia \\ Ismaili Alaoui \\ Nuclear Medicine Department, Oncology Hospital, University \\ Hospital Center Hassan II, Morocco
}

\begin{abstract}
Correspondence: Fabrice Fokoue, Nuclear Medicine Department, Oncology Hospital, University Hospital Center Hassan II, Sidi Mohamed Ben Abdellah of Fez, Morocco, Tel 212 0700561683, Email fabricefokoue2017@gmail.com
\end{abstract}

Received: August 26, 2020 | Published: September 15, 2020

\section{Introduction}

ONFH is a loss of vascularity to the femoral head leading to progressive collapse of bone and cartilage. It is more common in men (Sex-ratio 4/1). It can be primary, secondary or following medical or surgical treatment.

\section{Patient and method}

We report herein the case of a 45-year-old female patient who underwent osteosynthesis by double percutaneous screwing following a Garden III-classified left femoral neck fracture occurring during a public road accident. The evolution was marked 02 years later by the appearance of mechanical pain, progressive, with functional impotence localized in the left hip, crural irradiation. Standard pelvic-centered $x$-rays were negative, motivating the request of a bone scan looking for ONFH. The patient underwent a three-phase pelvic-centered bone scan after IV injection of $740 \mathrm{MBq}(20 \mathrm{mCi})$ of 99mTC-HMDP followed by a whole body scan and complementary pelvic-centered SPECT - CT with a Siemens gamma camera model Symbia T6 2010.

\section{Results}

The incidences carried out during the flow phase and the blood pool phase did not demonstrate a clearly individualizable perfusion or uptake anomaly regarding the two hips (Figure. 1). At the delayed phase, a unilateral hyperfixation was objectified to the left femoral head, the complement of which by SPECT - CT allowed an analysis of the sphericity of the femoral head with the possibility of staging of osteonecrosis in stage II according to the classification of Arlet and Ficat (Figure. 2). The patient subsequently underwent a total left hip arthroplasty with favorable outcome.
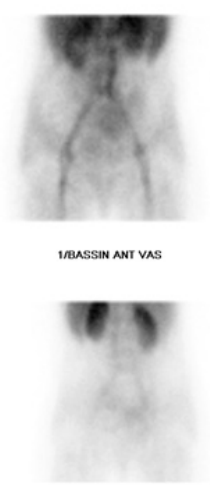

1.BASSIN POST VAS

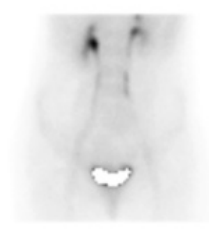

2renssin ANT IISS



2.18ASSIN POST nIS



1 BASSIN FACE ANT

D. BASSIN FACE POST |

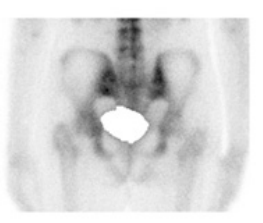

Figure I The flow or angiographic phase and the blood pool phase do not objectifying a clearly individualizable perfusion or uptake anomaly regarding the two hips. The delayed phase objectifying a unilateral hyperfixation on the left femoral head.

\section{Discussion}

ONFH is a disease process characterized by the cell death of osteocytes. It is the consequence of a mismatch between the oxygen needs of bone cells and the capacity of the local vascularization to meet these needs. Fractures are a frequent etiologic factor of osteonecrosis, in up to $50 \%$ of cases, especially osteonecrosis of the head of the femur. An intracapsular fracture can immediately alter the arterial vasculature of the femoral head via the intracapsular collateral vessels and epiphysites. The interruption of the vascularization, with occlusion, can be the direct consequence of an intra capsular fracture or the indirect consequence of a fracture causing an increase in the 
intra capsular pressure by hemarthrosis and / or bone displacement inside the inextensible capsule. Internal osteosynthesis can lead to additional vascular damage to small extra and intraosseous vessels and thus decrease the degree of bone vascularization. Osteonecrosis of the femoral head has been reported to occur in approximately $1 / 3$ of cases after fracture of the bone. Femoral neck osteonecrosis can occur in all types of femoral neck fractures, its frequency increases with Garden stages (it is more common in Garden types III and IV), but other factors also come into play in its genesis: importance of the initial displacement, rapidity of the operating time, quality of osteosynthesis.


Figure 2 SPECT-CT imaging showing a unilateral hyperfixation of the left femoral head corresponding to a heterogeneous density respecting the contours and the joint space in favor of osteonecrosis of the femoral head in stage II of Arlet and Ficat.

In about $80 \%$ of cases, ONFH appears within the first 2 years after the fracture, with onset 10 years or more after the fracture being exceptional. In the presence of internal osteosynthesis material, particularly surgical screws, the prevalence of ONFH is estimated between $2.5 \%$ and $18 \%$ according to various authors. The maximum latency period reported in the literature was 28 years. ${ }^{2,3}$ After internal osteosynthesis by screwing, the majority of patients have a favorable outcome. Apart from ONFH the other most common late complications are callus and pseudarthrosis. In addition, in our patient it was a Garden III classified femoral neck fracture and the onset of symptoms took place 02 years after surgery, this being consistent with the literature data and the postoperative complication found was ONFH. ${ }^{4}$ Clinically, ONFH may or may not remain asymptomatic. In symptomatic presentation, the pain is mechanical and permanent, increases with exertion and is aroused by internal rotation of the hip with progressive functional impotence. Lameness and limited internal rotation is present in all cases: this is the sign of the key described. Medical imaging is of primary importance in the diagnosis of ONTF. At the time of early diagnosis, standard radiographs are most often normal and this for several months, the length of this timeframe is not well known to date. The existence of a fine clarity "in eggshell" parallel to the subchondral zone or of a deformation, even minimal, of the cephalic contour are pathognomonic but already testify to a subchondral fracture, and therefore of 'a bad prognosis according to the Arlet and Ficat classification. ${ }^{5}$

Computed tomography (CT) scan, in the absence of MRI, detects changes in the bone pattern before conventional radiography: pre- radiological demineralization or loss of the sign of the asterisk characterized by disorganization of the central spans, or even their disappearance. The CT makes it possible to define an early osteonecrosis with a perfectly spherical head without subchondral dissection or conversely, an advanced osteonecrosis with subchondral dissection or depression of the sequestration. Magnetic Resonance Imaging (MRI) is the test of choice and gold standard for diagnosing ONFH. ${ }^{6}$ It shows the same classic images in T1 and T2 weighted sequences. Its interpretation is sometimes difficult due to the presence of old infarcts, the absence of fatty tissue or the presence of erythropoietic islets. Hence the need to search for the presence of joint effusion in T2-weighted images in the event of a recurrence or of a recent, progressive and symptomatic ONFH. However, the high cost and the unavailability of the exam in all centers can be an obstacle to diagnose most patients.

Bone scintigraphy is a sensitive but not very specific examination in the diagnosis of ONFH. The advent of hybrid imaging has significantly improved its diagnostic performance. ${ }^{7,8}$ In our patient, a three-phase bone scintigraphy and complement by SPECT CT made it possible to confirm ONFH and to stage the bone lesions thanks to the Arlet and Ficat classification, thus making it possible to evaluate the prognosis, thus orienting the therapeutic decision. In the weeks following the diagnosis of ONFH the patient underwent surgery by left hip arthroplasty with a favorable outcome.

\section{Conclusion}

This case confirms the significant role of SPECT-CT Imaging as Complement to a Three-Phase Bone Scintigraphy in the early diagnosis and prognosis of ONFH. Specifically in the Presence of Instrumentation after a Percutaneous Screwing osteosynthesis, thus allowing more optimal management.

\section{Acknowledgments}

None.

\section{Fundings}

None.

\section{Informed Consent}

Informed consent was obtained from the patient for being included in the study.

\section{Conflicts of interest}

Authors declare no conflict of Interest.

\section{References}

1. R Barnes, J T Brown, R S Garden, et al. Subcapital fractures of the femur. A prospective review. J Bone Joint Surg Br. 1976;58(1):2-24.

2. RS Garden. Stability and union in subcapital factures of the femur. $J$ Bone Joint Surg Br. 1964;46:630-647.

3. Kisielinski K, Niedhart C, Schneider U, et al. Osteonecrosis 15 years after femoral neck fracture and long-term low-dose inhaled corticosteroid therapy. Joint Bone Spine. 2004;71(3):237-239.

4. KE Nikolopoulos, SA Papadakis, KT Kateros, et al. Long-Term Outcome of Patients with Avascular Necrosis, after Internal Fixation of Femoral Neck Fractures. Injury. 2003;34(7):525-528.

5. P Ficat, J Arlet. Review of rheumatism and osteo-articular diseases. 1977;44(11):627-631. 
6. Nadja A Farshad-Amacker, Matthew F Koff, Jonathan P Dyke, et al Assessment of Osteonecrosis in the Presence of Instrumentation for Femoral Neck Fracture Using Contrast-Enhanced MAVRIC Sequence. HSS J. 2016;12(1):51-58.

7. F Paycha, A Girma, P Granier. Démarche sémiologique en TEMP/TDM osseuse. Une approche diagnostique intégrée de l'imagerie hybride. Médecine Nucléaire. 2010;34(8):490-511.
8. T Van den Wyngaert, K Strobel, WU Kampen, et al. The EANM practice guidelines for bone Scintigraphy. Eur J Nucl Med Mol Imaging. 2016;43:1723-1738. 